\title{
New Technology and New Shadow Play: New Media's Technical Promotion of Shadow Play
}

\author{
Lianghui $\operatorname{Jin}^{1 *}$ \\ ${ }^{1}$ Basic Department, School of Art, Jiangsu University Zhenjiang, Jiangsu, China
}

\begin{abstract}
Shadow Play is an ancient traditional folk art, which has been included in the Representative List of the Intangible Cultural Heritage of Humanity at present. Different forms of entertainment sources have appeared one after another with the rapid development of society. Because of its lack of competitiveness, Shadow Play has disappeared from the public's eye increasingly. In the meanwhile, different digital media technologies are used in a wide range of all aspects of life and entertainment. It's also a particular fine opportunity for Shadow Play to develop itself again. This article attempts to discuss the application of digital media art in the content and stage of Shadow Play and combine digital media art with the stage presentation of Shadow Play. There is some advice that is come up for the development of Shadow Play in the contemporary society, which also provides a better conservation for traditional art and hands it down.
\end{abstract}

\section{INTRODUCTION}

Shadow puppetry, also known as shadow play, is a kind of folk drama in which stories are performed by silhouettes of people made of animal skin or cardboard. During the performance, the artists manipulated the actors behind the white curtain while telling the story with local popular tunes, accompanied by percussion instruments and strings, which had a strong local flavor. The popular range is wide and the various shadow puppetry forms because of the different vocal cavity performed in different places. Shadow Play is a folk drama. And the animal skin or cardboard are used to shape the figure silhouette. It is performed under light on a white curtain. With the rapid development of society, this way of entertainment has been spread for a thousand years. However, other forms of entertainment by virtue of its colorful and more eye-catching characteristics have flooded people's lives, such as TV dramas, movies, TV shows and so on. And then they are continually developing and growing. There is no appeal to the public, due to single story, old, narrow audience of Shadow Play, which leads to fewer and fewer people are willing to learn and operate Shadow Play, and it will increasingly step down the history stage. It is extremely detrimental for the inheritance of Chinese traditional culture because of the decline of folk opera. But the development of various and different digital media technology provides many new and available science and technology for the redevelopment of Shadow Play. In a meanwhile, more possibility and uncertainty are injected into all kinds of traditional art performances and there is also a little contribution to protect inheritance of Shadow Play. This article discusses the application of digital media art in
Shadow Play, which gets a better understanding for the culture of Shadow. It also provides a new thought and idea for the integration of Shadow Play and other science and technology. In addition, a certain basis for further development of the Shadow Play is also included.

\section{Application of digital media art in the content of Shadow Play}

\subsection{Integration of artificial intelligence and Shadow Play}

Artificial intelligence (AI) is a branch of design research, design and application of intelligent machines in computer science or intelligent technology. It is a multidisciplinary technology integrating psychological cognition, machine learning, emotion recognition, human-computer interaction, data preservation and decision making. Shadow puppetry is a series of actions manipulated by a craftsman to tell a story to the public. There are technologies in ARTIFICIAL intelligence that can be linked to it. Artificial intelligence (AI) is a new and latest technical science. And it focuses on theories, methods, technologies and application systems applying for simulation, expansion and human intelligence's extension. A craftsman makes and manipulates a series of actions to shape Shadow Play so that can be told to the public. There are some close connections with Artificial Intelligence in technology. Initially, taking advantage of Blender (digital animation software) to digitally sculpt the Shadow Play, which attempts to restore the vivid and lively Shadow Play's image. And then, animation motion capture technology can be applied. Animation motion 
capture technology (also known as motion-capture) is usually focuses on the field of film production and game development to record human movements and convert them into digital model motion. Movements of Shadow Play are captured through technology of Mtheixamo(3D animation creation platform), importing the captured data into Blender afterwards. And then match the captured movements with the previously created Shadow Play through MakeHuman plugin. At the end, take advantage of After Effect to convert the video into a smooth mode [1]. In the future, Artificial Intelligence will flood widely in our life, and the integration of AI and Shadow Play will be a new art mode, which can both satisfies the public's interest and enthusiasm to new things and protects and inherits China's traditional folk Shadow Play. This integration approach not only applies for Shadow Play, but also applies for other traditional operas, has great application potential in entertainment.

\subsection{The integration of animated images and Shadow Play}

With China's reform and opening up and the rapid development of media communication technology, animated images have become one of the most dynamic visual patterns in contemporary society. Taking this as the background, a large scale subculture group of cartoons, animation and cartoons has formed in China with teenagers as the main body. As an experiencing activity, animation brings the audience different visual pleasure and emotional experiences through its rich visual expression. As a kind of communication activity, through watching behavior, the audience can understand the cultural information in the easiest and most interesting way in the shortest time. As an economic activity, it has already formed a huge cultural industry. With the rapid development of modern technology, animated images are produced accordingly. Once played and shown, they have gotten extremely praise from the public, especially among children. And it is also popular for Shadow Play among children in the past. At this point, there is the same audience so that is very fit for mutual integration and development. Some of the initial animation designs likewise involve some Shadow Play elements. Hence another way of performance of the Shadow Play will appear because of the integration of them [2].

The characters and colors in the animation are more distinct and conspicuous because of the integration of Shadow Play and two-dimensional animation. Meanwhile, the movements will be smoother than the Shadow Play. There are colorful images that easily attract the attention of children and make the public to remember easily by using Flash animation and other technology to combine Shadow Play with twodimensional animations. Simultaneously, in contemporary society, three-dimensional animation is becoming more and more widespread and popular. Some soft wares are used such as 3DS MAX and MAYA to shape and perform figures of Shadow Play. The combine of it and Shadow Play, which appears a different performance method of traditional Shadow Play and improves the sense of experience and interaction based on a two-dimension. Enhance the sense of experience and interaction. This integration of Shadow Play and animated images is consistent with the inherent performance mode of Shadow Play. And only in this way can it better enhance the Shadow Play's visibility. In the process of designing an animation, there are also some of the lens methods of movies can be used to make the animation plots of the Shadow Play rich and technical processing smooth.

\subsection{Integration of interactive technology and Shadow Play}

Interactive technology refers to the transmission and exchange of information, information, data and technical knowledge between nature and society. It uses certain means to achieve the purpose of interaction and gradually steps into the era of multi-field application. It defines the content and structure of communication between two or more interacting individuals so that they can cooperate with each other to achieve a common purpose. Interaction technology is the design field that defines and designs the behavior of artificial systems. The definition is about the content and structure of the communication between two or more interactive individuals so that they can cooperate with each other to achieve a certain purpose. It refers to the interaction between Shadow Play and the audience when interactive technology combines with Shadow Play's content. For instance, we can make a game according to Shadow Play's mode and form so that increase interactive options for the public and immerse it. Because of the advantage of games' wide audience, we can better promote the traditional Chinese opera Shadow Play. In addition, the Shadow Play can be made into an interactive video where the public can decide the direction of the story in terms of their idea. This sense of participation can attracts the public remain thoroughly in the Shadow Play. In addition, another AR (Augmented Reality) under the interactive technology can be used to promote the sense of experience for the public. For instance, design some three-dimensional picture books that present the figures of the Shadow Play, which both promotes the readability and enables the public to better understand the Shadow Play.

In the domestic, there is a digital museum of Shadow Play that partly takes advantage of some interactive technology attract more people to visit, which satisfy the requirements of professional talents, as well makes the general public have a certain understanding of Shadow Play[3]. In a meanwhile, there are a lot of digital media technologies that can be applied in museums. For instance, some large projection screens or other animations can be used to guide visitors. These interesting ways can increase a bit of interest to museums. 


\section{The application of digital media art in Shadow Play stage}

\subsection{The application of virtual reality technology in Shadow Play stage}

So-called "virtual reality", in essence is a kind of immersion, interactive experience, working experience in manufacturing this way may be cognitive, art, technology, or is the combination of all three, as long as this kind of experience can be within a certain range to be confused with reality, it can be broadly called "virtual reality"." Virtual reality" in the narrow sense refers to the experience of virtual reality produced by digital technology. When we say "virtual reality", we usually use it to refer to virtual reality in the narrow sense. Virtual reality is a digital environment with computer technology as its core, which is highly similar to the real environment in terms of vision, listening and touching. Users interact with the objects in the virtual environment with the related devices, and then produce the feeling and experience of the real environment. This technology has the following three characteristics: immersive, interactive and imaginative. It is three characteristics that attract more and more people to experience this technology. Applying this technology to the Shadow Play stage, a complete Shadow Play can be presented in front of our eyes through our simple gesture manipulation, which can greatly strengthen the audience's sense of interaction, let the audience become the Shadow Play operator, improve the audience's interest, so as to greatly promote the Shadow Play. At the same time, this technology can also make the audience's vision, touching and listening feel, all immersed in Shadow Play performance [4]. This kind of interactive feeling cannot be replicated by ordinary performance. Through this self-operated interaction, the public can more easily accept the traditional entertainment way of Shadow Play. In other words, virtual reality technology is a branch of interactive technology. They can complete the public's sense of experience. In the aspect of Shadow Play's inheritance and protection, it is very important for the public to have a strong sense of experience.

\subsection{The application of digital image technology in Shadow Play stage}

Traditional Shadow Play does not need complicated and changeable stage, only a curtain. In the past, this form of performance is an extremely big advantage. It can be performed without a venue and has strong mobility. This greatly saves the cost of craftsmen and facilitates them to perform in different places, thus improving their income. But for the ever-changing present, this kind of performance is too simple. There is no exquisite stage, gorgeous lighting effect, so it cannot attract the public. For the stage effect, performance methods, and public feelings, a delicate and perfect stage according to the performance theme can provide a lot of fun to an art performance, and can enhance the audience's sense of experience as well.
In Shadow Play performance, through a series of digital image technology, the stage setting is built into a three-dimensional background, and along with the content and plot of the Shadow Play, the lights and stage lighting images are arranged into related scenes. It is no longer a monotonous white curtain. The ups and downs of the story and the rendering of the surrounding environment give the audience a strong visual impact, which can better let the audience more immersive, and stimulate the audience's mood. So as to increase the audience of Shadow Play, let more people understand and inherit Shadow Play.

In Shadow Play stage performance, holographic projection can also be used. This technology usually appears in concerts, large-scale opening ceremonies and so on, which brings great shock to the public. In another way of thinking, Shadow Play is also a kind of projection technology. It projects the characters in various stories on the screen, and tells a complete and interesting story through flexible manipulation.

\subsection{The application of digital audio technology in shadow puppets stage}

The traditional Shadow Play performance, on the one hand, depends on the craftsman's manual operation of the Shadow Play to present the characters' every move; on the other hand, it relies on the craftsman's voice or some musical instruments to bring explanation to the Shadow Play. Through the combination of these two aspects, a vivid shadow puppets stage is presented.

At present, with the rapid development of various science and technology, there are better ways to replace the craftsman's oral skills. Through audio synthesis and other technologies, combined with the standard image of shadow puppets, Shadow Play becomes more attractive. In addition, traditional musical instruments and opera tunes have been unable to arouse the public's interest. The reasonable use of digital audio technology in Shadow Play can not only satisfy the requirements of the younger generation, but also make it more vivid and interesting [5]. The integration of digital audio technology makes Shadow Play more colorful, no longer single and boring, but also strengthens the rhythm of Shadow Play, and makes the public have a more pleasant mood when watching, which is more beneficial to the inheritance of Shadow Play.

\section{The future prospects}

The gradual decline of shadow puppetry is mainly due to its backwardness and backwardness, which seriously deviates from the trend of The Times and fails to meet people's growing cultural and aesthetic needs. Therefore, it is necessary to constantly innovate and study the integration of existing technologies into the shadow puppetry and enrich its transmission carriers and channels by using modern information technology. But at the same time, we should also make some innovations in the original content of shadow puppetry, add some modern popular elements, keep pace with The Times, 
and constantly increase the audience scope. While retaining the traditional cultural characteristics of shadow puppetry, we should also integrate new digital media technologies. There are more and more technologies can be utilized because of the rapid development of the society. And the existing technologies can also be more completely integrated into Shadow Play. At the same time, we should also make some innovations on the original content of Shadow Play, add some modern popular elements, keep up with the age, and gradually promote the range of audience. While retaining the traditional cultural characteristics of Shadow Play, we should also add and develop new digital media technologies. The integration of them can promote the competitiveness of Shadow Play in entertainment. Only in this way can more people know and like Shadow Play. The quality of Shadow Play will be extremely high and the visual experience will be better through such strict self-requirements so that the Shadow Play have better development in the future.

\section{Epilogue}

Shadow puppetry has become an integral part of the intangible cultural heritage with its unique production and performance. After more than one hundred years of development, it has become an independent and selfcontained type of art, which is a true description of the wisdom and life of the Chinese working people, contains the unique cultural customs of the Chinese nation, and has a high research value. To protect, inherit and develop shadow puppetry is an important part of China's socialist cultural construction. The time and society is developing rapidly, which makes the public audience lose interest in many traditional opera culture gradually. On the other hand, it also appears another opportunity for the development of folk traditional art. There is a mutual integration both digital media art and Shadow Play. It adopts the Artificial Intelligence, animation image, interactive technology in the Shadow Play content and uses virtual reality technology, digital image technology, and digital audio technology in the Shadow Play stage. At present what we need to do is that explore more technology and better method of ingratiation. Only in these ways can we inherit and protect the traditional folk art by our efforts such as Shadow Play in the future.

\section{REFERENCES}

1. Wang Jin. (2020) Activation and Reconstruction of Shadow Play in Animation Creation in Artificial Intelligence Era[J].Art and Design (theory), 420:6971

2. Wu Jing.The Research on Protection and Expansion of Digital Media to Shadow Art[C]. Nanjing University of the Arts, 2017

3. Yang Han.The Research on the Applied Design of Shanxi Shadow Art in New Media Interactive Experience Platform[C]. Xi'an University of Architecture and Technology,2020
4. Li Ruiyu. Research on the Application of Multimedia Technology in Shadow Play Art Display[C]. Hebei University of Science and Technology, 2020

5. Xue Yifan. Research on the Application of Multimedia Technology in Shadow Play Art Display[C]. Hebei University of Science and Technology, 2020 